\title{
Application of Ionic Liquid based Microwave-Assisted Extraction of the Secondary Metabolite from Peperomia pellucida (L) Kunth
}

\author{
Islamudin Ahmad ${ }^{1,2}$, Arry Yanuar ${ }^{1}$, Kamarza Mulia $^{3}$, Abdul Mun'im $^{1 *}$
}

Islamudin Ahmad ${ }^{1,2}$, Arry

Yanuar ${ }^{1}$, Kamarza Mulia ${ }^{3}$, Abdul

Mun'im ${ }^{1 *}$

'Department of Pharmacognosy-

Phytochemistry, Faculty of Pharmacy,

Universitas Indonesia, Depok, West

Java, INDONESIA.

2Department of Pharmaceutical Sciences, Faculty of Pharmacy, Mulawarman

University, Samarinda, East Kalimantan, INDONESIA.

${ }^{3}$ Department of Chemical Engineering, Faculty of Engineering, Universitas Indonesia, Depok, West Java, INDONESIA.

\section{Correspondence}

\section{Abdul Mun'im}

Faculty of Pharmacy, Universitas Indonesia, Gedung A Rumpun IImu Kesehatan Lantai 1, Kampus UI, Depok, Jawa Barat - 16424, INDONESIA.

Phone: +6221-7270031

E-mail: munim@farmasi.ui.ac.id

\section{History}

- Submission Date: 21-12-2016;

- Review completed: 05-01-2017;

- Accepted Date: 13-01-2017.

\section{DOI : 10.5530/pj.2017.2.38}

Article Available online http://www.phcogj.com/v9/i2

\section{Copyright}

(C) 2017 Phcog.Net. This is an openaccess article distributed under the terms of the Creative Commons Attribution 4.0 International license.

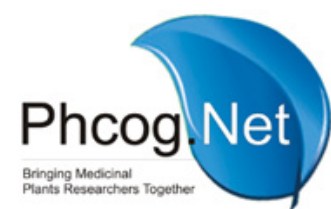

\begin{abstract}
Introduction: An ionic liquid (ILs) is a new alternative solvent (containing cations and anions) has the physical and specific solutions properties, and also had proven to be a promising substituent of conventional organic solvents are flammable, volatile and toxic in various processes. The ILS application in separating the secondary metabolites compound from Peperomia pellucida (L) Kunth herbaceous can be carried out using microwaveassisted extraction method (MAE). Objective: The aim of this study was to investigate the effect of ionic liquid based microwave-assisted extraction method for attracting the secondary metabolites compound from $P$. pellucida compared with a conventional solvent for the enrichment of secondary metabolites (especially polyphenols group) and their HPTLC fingerprinting profile. Methods: The herb dried powder extracted by microwave-assisted extraction using an ionic liquid such us 1-Butyl-3-methylimidazolium chloride ([BMIM]Cl), 1-Butyl-3-methylimidazolium Bromide ([BMIM]Br). The obtained extract solution was partitioned using an organic solvent; the extract was evaporated to obtain a dry extract and determination the HPTLC fingerprinting profile. Results: Based on the research results was obtained from the various types of solvents there are differences in the extraction of secondary metabolite.

Key words: HPTLC profile, Ionic liquid, Microwave-assisted extraction, Peperomia pellucida (L) Kunth, Secondary metabolite.
\end{abstract}

\section{INTRODUCTION}

Applications of ionic liquid in exploring the potential and content of the active compounds from natural materials continue to rise. ${ }^{1}$ Selection of an ionic liquid solvent with the approach to the application of the principle of Green Chemistry can use because it has the flexibility of a combination of ions to adjust the physicochemical properties of the target compound. Moreover, can consider as the constituents potential to replace organic solvents that are flammable, volatile and toxic while solvent ionic liquid has the opposite properties. ${ }^{2}$ Some previous study has been applied an ionic liquid for extraction of the target compound from plants such us trans-resveratrol from Polgonum cuspidatum Sieb. et. Zucc. (using 1-butyl-3-methyl-imidazolium bromide [BMIM] $\mathrm{Br}) .^{3}$ Alkaloid phenolic (Liensinine, Isoliensinine, and Neferine) from Nelumbo nucifera Graerthn (using 1-butyl-3-methylimidazolium tetrafluoroborate [BMIM] $\left.\mathrm{BF}_{4}\right) .{ }^{4}$ Piperine from Piper ningrum (using $\left.[\mathrm{BMIM}] \mathrm{BF}_{4}\right) .{ }^{5}$ Luteolin and Apigenin from Apium graveolens (using 1-buthyl-3methylimidazolium methyl sulfate $\left.[\mathrm{BMIM}] \mathrm{C}_{1} \mathrm{OSO}_{3}\right) .{ }^{6}$ Tannin from Galla chinensis (using [BMIM]Br), ${ }^{7}$ phenolic compound from Pyrola calliandra $\mathrm{H}$. Andr. (using [BMIM] $\mathrm{BF}_{4}$ ), ${ }^{8}$ isoflavones from Radix pueraria (using [BMIM]Br), ${ }^{9}$ Flavanoids from Scutellaria baicalein Georgi (using 1-octyl-3-methylimidazolium bromide [OMIM] $\mathrm{Br}),{ }^{10}$ and Gallic acid from Suaeda glauca Bge. (us- ing 1-hexyl-3-methylimidazolium chloride [HMIM] Cl). ${ }^{11}$

Suruhan (Peperomia pellucida (L.) Kunth) herbs is widely used in traditional medicine and known as "Jamu". The aerial part is used traditionally for the treatment of a headache accompanied by fever, abdominal pain, diabetes mellitus, hyperuricemia, and hypertension. ${ }^{12,13}$ These herbs contain various chemical constituent such as amino acid, protein, carbohydrate, minerals, tannin, saponin, phenolic, steroid, terpenoid, dan alkaloid. ${ }^{14-20}$ Some compound reported from this plant, including secolignan, tetrahydrofuran, peperomins, sesamin, isoswesitin, ${ }^{21}$ apiole, ${ }^{22}$ patuloside A (Xanthone), ${ }^{23}$ dillapiole, ${ }^{24}$ chromene, ${ }^{25}$ and quercetin. ${ }^{26}$ In isolating compounds such as the above are very tough to replicate and requires many samples to be able to isolate it. Because the yield of this plant, both in the form of dried simplicia and crude extracts are very low. Therefore, the application of ionic liquid conducted to obtain the maximum target compound. However, extraction methods using ionic liquid based microwave assisted extraction of Suruhan herbs have not reported. The objective of the study was the application of an ionic liquid solvent (based microwave assisted extraction) from this herbs. 


\section{MATERIAL AND METHODS}

\section{Herb Materials}

The herbs of Suruhan (Peperomia pellucida (L.) Kunth) were collected from Baras, North Mamuju, West Sulawesi, Indonesia, The voucher specimens were identified at the Herbarium Bogoriense, Bogor, West Java, Indonesia and deposited at Pharmacognosy and Phytochemistry Laboratory, Universitas Indonesia, Depok, West Java, Indonesia. The herbs washed and dried. The dried sample was powdered using a grinder.

\section{Chemical Materials and General Equipment}

The chemical and equipment utilized in this study, including aquadest, dichloromethane (pro analyze), n-hexane (pro analyze), and ethyl acetate (pro analyze) purchased from PT. Smart Lab Indonesia, Indonesia. 1-buthyl-3-methylimidazolium bromide ([BMIM]Br) and 1-buthyl3-methylimidazolium chloride ([BMIM]Cl) purchased from Shanghai Cheng Jie Chemical Co. LTD, China. Design Expert 10 licensed software (STATE-EASE, USA). TLC Silica Gel $60 \mathrm{~F}_{254}$ (Merck, Germany). Chamber (Camag, Switzerland), Rotary Evaporator (Buchi, Germany), Modena Microwave 900 Watt (Buono-MV3002), Vortex mixer (Stuart, Germany). Densitometer Camag Wincat 3 TLC Scanner (Camag, Switzerland), as well as equipment for maceration.

\section{Extraction Methods}

\section{Conventional Extraction Methods}

The dried herbs powder ( 3 grams) were macerated using n-hexane and ethyl acetate, respectively. ${ }^{27}$ The sample was macerated using $n$-hexane for 24 hours. Next, the residual was macerated again using ethyl acetate for 24 hours. Each the extracts solution was evaporated to obtain dried extracts and ready for analyzed by HPTLC.

\section{Non-Conventional Extraction Methods}

Extraction using ionic liquid based microwave-assisted extraction. ${ }^{28-31}$ Briefly, a 3 g sample was extracted using ionic liquid based microwaveassisted extraction with [BMIM] $\mathrm{Br}$ and [BMIM] $\mathrm{Cl}$ as a solvent. The experiments performed at several combination such us ionic liquid concentration $(0.6,0.8,1,1.2$, and $1.4 \mathrm{~mol} / \mathrm{L})$, ratio liquids/solid $(8,10$, 12,14 , and $16 \mathrm{ml} / \mathrm{g}$ ), microwave power $(10 \%, 30 \%, 50 \%, 70 \%$, and $100 \%)$, and extraction time (2, 3, 4, 5, and 6 minutes) using Design Expert 10 (STATEASE-USA). After the extraction process, a mixture/raw extracts filtered with cotton and the filtrate was cooled to room temperature. Next, the filtrate was partitioned using liquid-liquid extraction (with the addition of ethyl acetate solvent) and was stirred using vortex mixer for 10 minutes then put to centrifugation at $4500 \mathrm{tr} \mathrm{min}^{-1}$ during $15 \mathrm{~min}$ utes. Both solution layers were separated, the ethyl acetate layer which contains some metabolites was evaporated to obtain dried extracts. The final extracts (Figure 1) were prepared in ethyl acetate, were analyzed by HPTLC.

\section{Determination of HPTLC Fingerprinting Profile}

For determination of HPTLC fingerprinting profile is conducted using dichloromethane: ethyl acetate with comparison 3:7 as the mobile phase. Precoated silica gel $\mathrm{GF}_{254}$ (Merck, Germany) as stationary phase was activated, and detection using densitometer Camag Wincat 3 TLC Scanner (Camag, Switzerland) at daylight, short ultraviolet $254 \mathrm{~nm}$, long ultraviolet $366 \mathrm{~nm}$.

\section{RESULTS AND DISCUSSION}

\section{Ionic Liquid based Microwave-Assisted Extraction (IL-MAE)}

The usage of ILS solvent has conducted over the last decade. Various techniques, cost, and complexity have done to can be applied to extract the active components of the plant. Ideally, the extraction method selected must a complete to produce to the maximum desired component of secondary metabolites, with fixed should give priority to the principle is simple, fast, safe, economical, environmentally friendly, and can be reproduced. The Heat Reflux Extraction (HRE) is the classic method is the most appropriate use than other classical methods. However, the use of high-tech extraction methods has proven to be more efficient and economical for extracting the active components of the plant by using microwave-assisted extraction (IL-MAE). Some of the experimental factors employed in the extraction method using Design Expert 10 namely time of extraction, microwave power, ILs concentration, and ratio liquid/ solid. The extraction time factors have an influence on duration of the sample mixture exposed to microwave radiation. The microwave power factors have the effect of power and temperature of a microwave. ILs concentration The ILs concentration associated with viscosity mainly to penetrate and damage the plant cell walls. The ratio of liquid/solid associated a balance and saturation between the solid phase and the liquid phase in the mixture sample. For the phases of the extraction process and ILs recovery can be seen in Figure 1.

\section{HPTLC Fingerprinting Profile}

Each extract obtained from conventional methods, and non-conventional methods were spotted on TLC plate $(10 \times 20 \mathrm{~cm})$ and were eluted using dichloromethane: ethyl acetate (3:7) eluent, then was analyzed using densitometer at $254 \mathrm{~nm}$ dan $366 \mathrm{~nm}$ wavelength to confirm the presence of various phytoconstituents in the each extract.

HPTLC fingerprinting of conventional extraction methods of P.pellucida extract showed a total of 14 peaks at different $\mathrm{Rf}$ values and peak area at $254 \mathrm{~nm}$ whereas six peaks were observed in HPTLC chromatogram at $366 \mathrm{~nm}$ from $\mathrm{n}$-hexane extract (Figure 2; Extract A). HPTLC fingerprinting for ethyl acetate extract (Figure 2; Extract B) showed a total of 11 peaks at different $\mathrm{Rf}$ values and peak area at $254 \mathrm{~nm}$ and a total of 8 peaks at $366 \mathrm{~nm}$. Moreover, directly ethyl acetate extract showed a total of 8 peaks at different $\mathrm{Rf}$ values and peak area at $254 \mathrm{~nm}$ and a total of 7 peaks at $366 \mathrm{~nm}$ (Figure 2; Extract C). The total number of constituents (No. of Peaks) in the each extract and their Rf summarized in Table 1. Based on the results of HPLC fingerprinting profile indicates different compounds was attracted in an extract from extraction process using

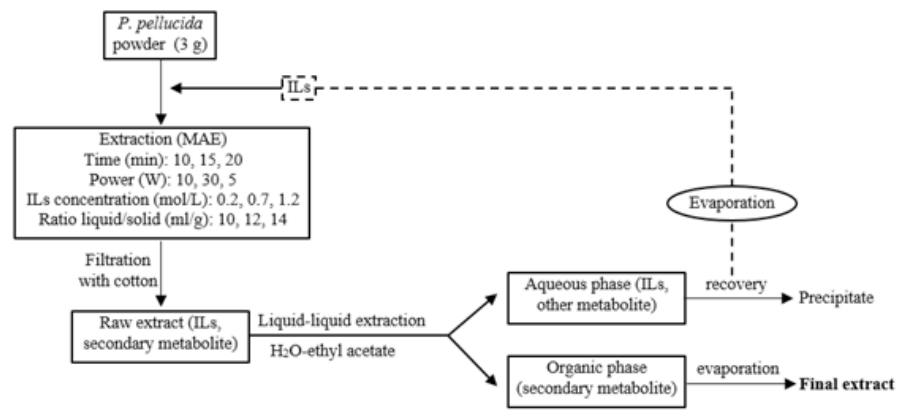

Figure 1. The extraction process using lonic Liquid Based Microwave-assisted Extraction (IL-MAE) of P. pellucida, recovery of secondary metabolites and possible regeneration of ionic liquid solvent (ILs). 

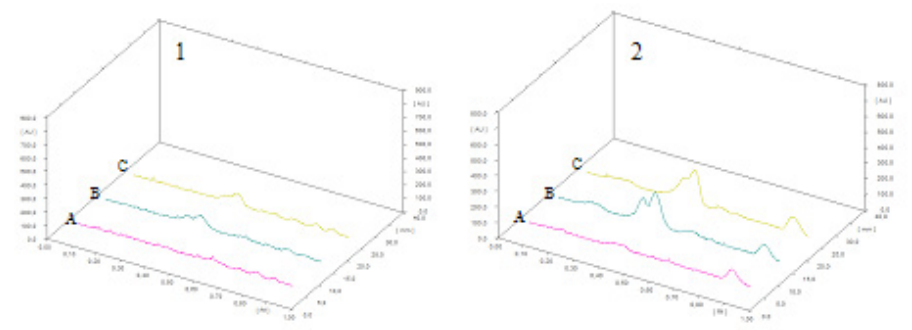

Figure 2. Chromatogram of the extract of $P$. pellucida using conventional extraction ( $\mathrm{A}$; $\mathrm{n}$-hexane extract, $\mathrm{B}$; ethyl acetate extract, $\mathrm{C}$; directly ethyl acetate extract).
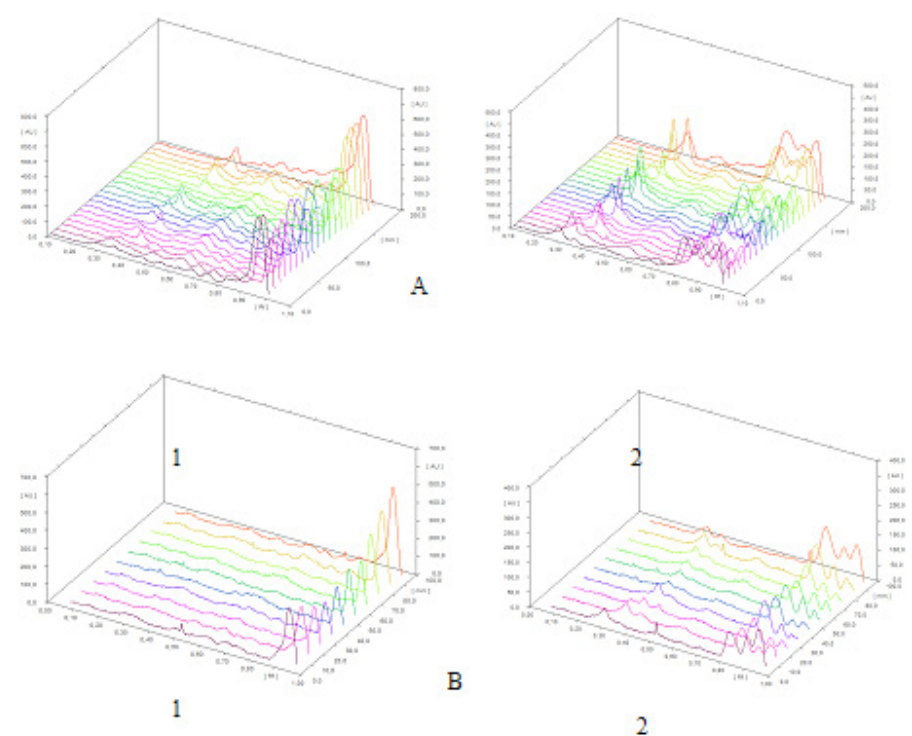

2

Figure 3. Chromatogram of the extract of P. pellucida using lonic Liquid Based Microwave-Assisted Extraction (IL-MAE with [BMIM]Cl as solvent) at $254 \mathrm{~nm}$ (1) and $366 \mathrm{~nm}$ (2) wavelength. Where A; 1-20 extract, B; 21-30 extract.
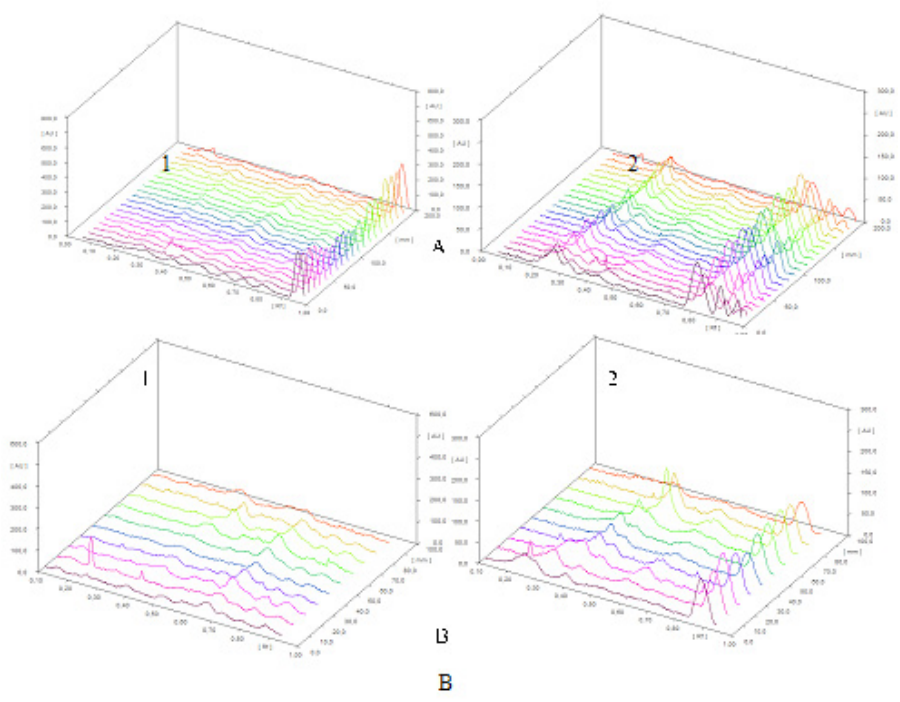

Figure 4. Chromatogram of the extract of P. pellucida using lonic Liquid Based Microwave-Assisted Extraction (IL-MAE with [BMIM]Br as solvent) at $254 \mathrm{~nm}$ (1) and $366 \mathrm{~nm}$ (2) wavelength. Where $A ; 1-20$ extract, $B ; 21-30$ extract.
Table 1: HPTLC fingerprinting profile of $P$. pellucida using conventional extraction ( $A$; $n$-hexane extract, B; ethyl acetate extract, C; directly ethyl acetate extract)

\begin{tabular}{|c|c|c|c|c|}
\hline Extract & Wavelength & $\begin{array}{l}\text { No. of } \\
\text { Peaks }\end{array}$ & Rf Values & $\begin{array}{c}\text { Percentage peak } \\
\text { area }\end{array}$ \\
\hline \multirow[t]{2}{*}{ A } & $254 \mathrm{~nm}$ & 14 & $\begin{array}{c}0.10,0.12,0.16 \\
0.21,0.36,0.40 \\
0.45,0.51,0.56 \\
0.58,0.64,0.69 \\
\quad 0.75,0.83\end{array}$ & $\begin{array}{c}3.26,3.68,7.04, \\
2.58,9.46,8.29 \\
3.50,9.09,7.46, \\
9.09,16.23,1.71 \\
5.41,14.88,7.39\end{array}$ \\
\hline & $366 \mathrm{~nm}$ & 6 & $\begin{array}{c}0.16,0.34,0.40 \\
0.51,0.58,0.80\end{array}$ & $\begin{array}{c}5.89,11.35,15.18 \\
2.05,4.89\end{array}$ \\
\hline \multirow[t]{2}{*}{ B } & $254 \mathrm{~nm}$ & 11 & $\begin{array}{c}0.15,0.19,0.32 \\
0.34,0.40,0.50 \\
0.58,0.60,0.69 \\
0,76,0,84\end{array}$ & $\begin{array}{c}2.50,2.22,4.19 \\
21.22,43.69,1.01 \\
3.22,7.52,2.27 \\
8.25,3.89\end{array}$ \\
\hline & $366 \mathrm{~nm}$ & 8 & $\begin{array}{c}0.14,0.20,0.31 \\
0.40,0.54,0.64 \\
0.69,0.82\end{array}$ & $\begin{array}{c}4.88,2.50,28.70 \\
40.14,6.54,1.20 \\
0.28,15.77\end{array}$ \\
\hline \multirow{2}{*}{ C } & $254 \mathrm{~nm}$ & 8 & $\begin{array}{c}0.11,0.40,0.46 \\
0.55,0.62,0.66 \\
0.76,0.83\end{array}$ & $\begin{array}{c}2.54,37.85,28.83 \\
2.36,3.50,4.44 \\
11.44,9.04\end{array}$ \\
\hline & $366 \mathrm{~nm}$ & 7 & $\begin{array}{c}0.11,0.14,0.18 \\
0.35,0.53,0.62,0.81\end{array}$ & $\begin{array}{c}0.94,3.35,4.02 \\
67.80,2.40,1.56 \\
19.93\end{array}$ \\
\hline
\end{tabular}

different solvents. For example, chlorophyll compounds contained in the sample mostly attracted in the n-hexane.

The HPTLC fingerprinting of non-conventional extraction using [BMIM] $\mathrm{Cl}$ as solvent of each extract, showed a total of successively 8 , $6,8,9,8,8,6,8,11,8,10,11,8,9,8,6,8,9,8,7,9,9,9,11,8,6,8,9,10$, and 9 peaks at different Rf values. Moreover, peak area at $254 \mathrm{~nm}$ with a total of $7,7,7,6,6,6,4,6,7,6,8,7,7,5,5,4,5,5,5,7,3,6,8,6,3,5,5$, 4,5 , and 4 peaks were observed at $366 \mathrm{~nm}$, respectively. The total number of peaks in the extract and their Rf is summarized in Table 2, and chromatographic profile had shown in Figure 3. Whilst the HPTLC fingerprinting of each extract using non-conventional extraction method with [BMIM] $\mathrm{Br}$ as a solvent showed a total of $8,9,12,9,10,8,9,9,8$, $10,8,8,5,7,8,13,9,9,12,8,10,8,9,9,12,12,10,10,13$, and 10 peaks were observed at $254 \mathrm{~nm}$ and a total of $5,6,6,5,4,6,3,5,5,3,4,5,4,4$, $5,5,5,6,5,5,5,6,8,7,7,7,8,7$, and 5 peaks were observed at $366 \mathrm{~nm}$, respectively. The total number of peaks in the extract and their Rf summarized in Table 3 and chromatographic profile had shown in Figure 4. The results of HPTLC fingerprinting profile indicate the effect of the usage of ionic liquid as a solvent and experiment factors (such as the time of extraction, microwave power, Ionic Liquid Solvent (ILs) concentration, and solid/liquid ratio).

The results of this study are the first step to conducting further research towards optimization of extraction methods to the isolation of biomarker compounds from this herbs.

\section{CONCLUSION}

From the above results indicate that the usage of ionic liquids as solvents can apply to extracting secondary metabolites from $P$. pellucida herbs (especially if it has been known that the desired target compound). So to perform extraction becomes more quickly, easily, and efficiently than conventional methods in general. 
Table 2: HPTLC finger printing profile of $P$. pellucida using lonic Liquid Based Microwave-Assisted Extraction (IL-MAE with [BMIM]CI as solvent)

\begin{tabular}{|c|c|c|c|c|}
\hline Extract & Wavelength & $\begin{array}{l}\text { No. of } \\
\text { Peaks }\end{array}$ & Rf Values & Percentage peak area \\
\hline \multirow{2}{*}{1} & $254 \mathrm{~nm}$ & 8 & $0.26,0.33,0.53,0.61,0.66,0.72,0.74,0.79$ & $14.40,22.25,9.90,5.41,17.59,5.40,13.21$ \\
\hline & $366 \mathrm{~nm}$ & 7 & $0.25,0.34,0.59,0.66,0.72,0.77,0.87$ & $9.91,2.53,1.01,3.78,1.37,45.97,35.42$ \\
\hline \multirow{2}{*}{2} & $254 \mathrm{~nm}$ & 6 & $0.28,0.47,0.58,0.66,0.72,0.79$ & $27.94,11.79,25.02,11.93,8.11,15.22$ \\
\hline & $366 \mathrm{~nm}$ & 7 & $0.27,0,37,0.53,0.67,0.70,0.78,0.88$ & $23.45,4.86,6.46,1.10,1.04,44.00,19.11$ \\
\hline \multirow{2}{*}{3} & $254 \mathrm{~nm}$ & 8 & $0.23,0.30,0.44,0.48,0.59,0.68,0.73,0.80$ & $1.64,17.20,3.03,10.69,36.33,5.61,6.40,19.09$ \\
\hline & $366 \mathrm{~nm}$ & 7 & $0.27,0.37,0.44,0.52,0.57,0.80,0.89$ & $23.59,7.07,1.46,2.93,8.27,38.90,17.78$ \\
\hline \multirow{2}{*}{4} & $254 \mathrm{~nm}$ & 9 & $0.28,0.38,0.45,0.49,0.56,0.60,0.67,0.73,0.80$ & $6.07,21.12,2.01,5.11,8.02,22.05,13.23,13.23,12.48,9.91$ \\
\hline & $366 \mathrm{~nm}$ & 6 & $0.36,0.54,0.61,0.78,0.88,0.95$ & $23.45,3.96,3.07,40.49,18.54,10.49$ \\
\hline \multirow{2}{*}{5} & $254 \mathrm{~nm}$ & 8 & $0.31,0.41,0.48,0.58,0.68,0.73,0.80,0.86$ & $20.45,4.22,11.12,35.16,8.10,7.07,10.97,2.90$ \\
\hline & $366 \mathrm{~nm}$ & 6 & $0.30,0.40,0.53,0.62,0.79,0.89$ & $31.28,7.34,4.20,3.17,44.35,9.65$ \\
\hline \multirow{2}{*}{6} & $254 \mathrm{~nm}$ & 8 & $0.27,0.32,0.40,0.48,0.59,0.67,0.74,0.80$ & $1.21,12.46,3.45,10.49,44.12,6.97,4.25,17.06$ \\
\hline & $366 \mathrm{~nm}$ & 6 & $0.29,0.38,0.53,0.61,0.79,0.89$ & $25.39,7.19,5.48,6.39,46.71,8.85$ \\
\hline \multirow{2}{*}{7} & $254 \mathrm{~nm}$ & 6 & $0.38,0.50,0.60,0.67,0.73,0.80$ & $27.32,11.66,29.23,14.41,5.25,12.13$ \\
\hline & $366 \mathrm{~nm}$ & 4 & $0.37,0.54,0.63, .078$ & $31.36,2.52,3.79,62,34$ \\
\hline \multirow{2}{*}{8} & $254 \mathrm{~nm}$ & 8 & $0.12,0.31,0.49,0.58,0.69,0.73,0.80,0.89$ & $3.27,18.24,12.81,36.95,5.07,8.24,12.23,3.20$ \\
\hline & $366 \mathrm{~nm}$ & 6 & $0.29,0.42,0.52,0.70,0.78,0.88$ & $28.36,2.48,4.08,0.60,45.56,18.91$ \\
\hline \multirow[t]{2}{*}{9} & $254 \mathrm{~nm}$ & 11 & $0.28,0.32,0.44,0.50,0.57,0.68,0.73,0.76,0.80,0.90,0.93$ & $\begin{array}{c}1.09,9.55,1.41,5.35,29.97,6.75,2.25,2,47,7.32,0.69 \\
37.13\end{array}$ \\
\hline & $366 \mathrm{~nm}$ & 7 & $0.30,0.39,0.53,0.60,0.79,0.89,0.90$ & $32.10,6.09,2.86,6.37,41.13,2.78 .8 .66$ \\
\hline \multirow{2}{*}{10} & $254 \mathrm{~nm}$ & 8 & $0.28,0.35,0.48,0.58,0.67,0.72,0.74,0.80$ & $3.26,18.60,14.01,32.08,9.04,2.60,7.58,12.82$ \\
\hline & $366 \mathrm{~nm}$ & 6 & $0.34,0.55,0.61,0.72,0.78,0.88$ & $26.35,3.65,4.52,0.53,40.66,24.29$ \\
\hline \multirow{2}{*}{11} & $254 \mathrm{~nm}$ & 10 & $0.14,0.28,0.37,0.51,0.59,0.68,0.74,0.76,0.81,0.84$ & $1.74,2.46,21.89,6.68,37.96,9.60,1.48,4.88,5.78,7.53$ \\
\hline & $366 \mathrm{~nm}$ & 8 & $0.36,0.55,0.60,0.74,0.76,0.78,0.90,0.95$ & $23.36,3.73,9.04,0.63,1.11,44.65,10.31,7.16$ \\
\hline \multirow[t]{2}{*}{12} & $254 \mathrm{~nm}$ & 11 & $0.27,0.32,0.34,0.49,0.51,0.56,0.61,0.67,0.73,0.79,0.85$ & $\begin{array}{c}1.73,0.56,25.37,1.25,8.83,10.61,19.35,9.06,6.25,11.41 \\
5.58\end{array}$ \\
\hline & $366 \mathrm{~nm}$ & 7 & $0.32,0.34,0.49,0.55,0.60,0.76,0.88$ & $0.57,32.83,0.54,2.81,7.02,7.02,43.88,13.34$ \\
\hline \multirow{2}{*}{13} & $254 \mathrm{~nm}$ & 8 & $0.29,0.32,0.38,0.50,0.59,0.70,0.73,0.81$ & $4.48,14.44,9.98,9.01,37.56,3.25,8.77,12.50$ \\
\hline & $366 \mathrm{~nm}$ & 7 & $0.31,0.38,0.43,0.60,0.62,0.80,0.89$ & $27.40,7.96,1.97,3.89,5.97,39.32,13.50$ \\
\hline \multirow{2}{*}{14} & $254 \mathrm{~nm}$ & 9 & $0.20,0.26,0.32,0.50,0.56,0.68,0.73,0.81,0.89$ & $3.79,2.63,9.80,9.73,38.77,6.66,11.11,13.37,4.13$ \\
\hline & $366 \mathrm{~nm}$ & 5 & $0.30,0.41,0.55,0.79,0.89$ & $20.81,2.36,9.98,45.33,21.52$ \\
\hline \multirow{2}{*}{15} & $254 \mathrm{~nm}$ & 8 & $0.12,0.38,0.46,0.50,0.60,0.72,0.80,0.89$ & $1.15,12.40,1.20,4.53,17.85,17.85,1.99,6.76,54,12$ \\
\hline & $366 \mathrm{~nm}$ & 5 & $0.38,0.53,0.60,0.79,0.88$ & $26.39,5.10,8.15,38.08,22.27$ \\
\hline \multirow{2}{*}{16} & $254 \mathrm{~nm}$ & 6 & $0.33,0.47,0.57,0.61,0.74,0.80$ & $20.30,16.27,13.09,21.46,9.61,19,28$ \\
\hline & $366 \mathrm{~nm}$ & 4 & $0.32,0.80,0.90,0.95$ & $18.50,43.41,11.74,26.35$ \\
\hline \multirow{2}{*}{17} & $254 \mathrm{~nm}$ & 8 & $0.35,0.46,0.50,0.55,0.60,0.67,0.72,0.78$ & $15.33,1.07,4.94,10.37,21.97,8.01,11.05,27.27$ \\
\hline & $366 \mathrm{~nm}$ & 5 & $0.36,0.53,0.61,0.76,0.88$ & $21.04,6.54,5.96,44.56,20.89$ \\
\hline \multirow{2}{*}{18} & $254 \mathrm{~nm}$ & 9 & $0.11,0.36,0.49,0.55,0.60,0.67,0.72,0.78,0.88$ & $0.44,5.67,2.49,3.69,9.93,4.16,5.00,12.12,56.50$ \\
\hline & $366 \mathrm{~nm}$ & 5 & $0.35,0.53,0.61,0.78,0.88$ & $13.23,9.70,32.28,20.14,24.65$ \\
\hline \multirow{2}{*}{19} & $254 \mathrm{~nm}$ & 8 & $0.40,0.49,0.55,0.60,0.67,0.72,0.78,0.86$ & $5.81,2.59,3.48,6.54,4.81,5.82,10.69,60.26$ \\
\hline & $366 \mathrm{~nm}$ & 5 & $0.38,0.53,0.77,0.88,0.93$ & $15.29,8.91,34.19,18.51,23.09$ \\
\hline \multirow{2}{*}{20} & $254 \mathrm{~nm}$ & 7 & $0.38,0.46,0.52,0.58,0.65,0.71,0.86$ & $7.06,3.70,4.59,9.39,7.13,9.34,58.78$ \\
\hline & $366 \mathrm{~nm}$ & 7 & $0.34,0.49,0.60,0.66,077,0.88,0.94$ & $12.54,5.76,2.20,3.08,38.41,19.78,18.24$ \\
\hline \multirow{2}{*}{21} & $254 \mathrm{~nm}$ & 9 & $0.12,0.22,0.36,0.48,0.55,0.63,0.70,0.76,0.84$ & $5.81,6.36,10.27,11.79,7.03,32,59,10.48,5.18,10.49$ \\
\hline & $366 \mathrm{~nm}$ & 3 & $0.19,0.36,0.82$ & $34.65,7.48,57.87$ \\
\hline
\end{tabular}


Table 2: Con

\begin{tabular}{|c|c|c|c|c|}
\hline \multirow{2}{*}{22} & $254 \mathrm{~nm}$ & 9 & $0.12,0.21,0.29,0.38,0.50,0.63,0.71,0.76,0.84$ & $5.95,19.69,5.17,6.36,9.81,28.87,6.05,9.14,8.93$ \\
\hline & $366 \mathrm{~nm}$ & 6 & $0.21,0.24,0.28,0.38,0.54,0.83$ & $5.94,5.20,19.57,5.40,5.76,58.12$ \\
\hline \multirow{2}{*}{23} & $254 \mathrm{~nm}$ & 9 & $0.12,0.28,0.36,0.41,0.51,0.58,0.64,0.75,0.82$ & $4.99,0.96,3.86,10.60,10.30,8.82,34.79,9.05,12.63$ \\
\hline & $366 \mathrm{~nm}$ & 8 & $0.24,0.27,0.34,0.43,0.56,0.60,0.78,0.82$ & $2.59,16.48,17.99,3.15,6.56,3.76,2.05,47.42$ \\
\hline \multirow[t]{2}{*}{24} & $254 \mathrm{~nm}$ & 11 & $0.13,0.23,0.37,0.40,0.43,0.52,0.58,0.63,0.72,0.76,0.83$ & $\begin{array}{c}4.34,3.39,5.12,8.52,6.84,3.40,11.54,38.56,5.01,6.57 \\
7.82\end{array}$ \\
\hline & $366 \mathrm{~nm}$ & 6 & $0.23,0.35,0.42,0.57,0.72,0.82$ & $1.93,23.33,9.03,3.04,49.64$ \\
\hline \multirow{2}{*}{25} & $254 \mathrm{~nm}$ & 8 & $0.36,0.40,0.52,0.64,0.72,0.76,0.83,0.86$ & $6.65,16.71,7.68,43.36,4.69,8.11,6.83,5.97$ \\
\hline & $366 \mathrm{~nm}$ & 3 & $0.35,0.43,0.83$ & $31.42,8.21,60.37$ \\
\hline \multirow{2}{*}{26} & $254 \mathrm{~nm}$ & 6 & $0.40,0.51,0.58,0.63,0.76,0.83$ & $10.51,6.57,9.69,53.22,9.29,10.72$ \\
\hline & $366 \mathrm{~nm}$ & 5 & $0.34,0.43,0.60,0.66,0.82$ & $24.03,5.08,9.84,5.41,55.65$ \\
\hline \multirow{2}{*}{27} & $254 \mathrm{~nm}$ & 8 & $0.36,0.52,0.58,0.63,0.75,0.77,0.83,0.85$ & $24.18 .7 .68,11.53,42.15,1.50,3.86,2.80,6.30$ \\
\hline & $366 \mathrm{~nm}$ & 5 & $0.37,0.43,0.58,0.65,0.82$ & $25.82,11.29,7.60,5.61,49.68$ \\
\hline \multirow{2}{*}{28} & $254 \mathrm{~nm}$ & 9 & $0.12,0.18,0.42,0.52,0.58,0.63,0.75,0.83,0.87$ & $2.67,1.50,32.95,5.14,6.11,33.46,7.34,6.37,4.46$ \\
\hline & $366 \mathrm{~nm}$ & 4 & $0.42,0.57,0.61,0.82$ & $40.04,5.18,12.68,42.11$ \\
\hline \multirow{2}{*}{29} & $254 \mathrm{~nm}$ & 10 & $0.18,0.22,0.28,0.35,0.43,0.49,0.57,0.64,0.75,0.82$ & $4.01,3.79,4.05,3.52,18.38,8.37,11.79,10.45,7.54$ \\
\hline & $366 \mathrm{~nm}$ & 5 & $0.35,0.41,0.57,0.61,0.81$ & $1.87,38.42,8.36,5.79,45.56$ \\
\hline \multirow{2}{*}{30} & $254 \mathrm{~nm}$ & 9 & $0.19,0.25,0.27,0.35,0.48,0.55,0.61,0.74,0.79$ & $4.02,2.75,4.85,38.32,4.4420 .06,22.78,1.81,0.98$ \\
\hline & $366 \mathrm{~nm}$ & 4 & $0.25,0.41,0.78,0.81$ & $6.37,13.42,2.54,77.67$ \\
\hline
\end{tabular}

Table 3: HPTLC fingerprinting profile of $P$. pellucida using lonic Liquid Based Microwave-Assisted Extraction (IL-MAE with [BMIM]Br as solvent

\begin{tabular}{|c|c|c|c|c|}
\hline Extract & Wavelength & $\begin{array}{l}\text { No. of } \\
\text { Peaks }\end{array}$ & Rf Values & Percentage peak area \\
\hline \multirow{2}{*}{1} & $254 \mathrm{~nm}$ & 8 & $0.13,0.22,0.29,0.40,0.49,0.62,0.69,0.78$ & $1.14,10.65,16.15,12.16,22.73,10.80,13,15$ \\
\hline & $366 \mathrm{~nm}$ & 5 & $0.20,0.31,0.41,0.76,0.87$ & $23.02,5.53,4.42,49.46,17.57$ \\
\hline \multirow{2}{*}{2} & $254 \mathrm{~nm}$ & 9 & $0.24,0.36,0.42,0.51,0.56,0.62,0.68,0.71,0.79$ & $16.65,3.20,18.96,9.42,11.89,9.99,4.03,11.09,14.78$ \\
\hline & $366 \mathrm{~nm}$ & 6 & $0.21,0.29,0.41,0.78,0.88,0.94$ & $26.40,6.65,10.50,45.50,8.50,2.40$ \\
\hline \multirow[t]{2}{*}{3} & $254 \mathrm{~nm}$ & 12 & $\begin{array}{c}0.11,0.14,0.18,0.24,0.35,0.41,0.44,0.53,0.64,0.70, \\
0.80,0.93\end{array}$ & $\begin{array}{c}1.54,1.32,1.41,4.57,11.87,4.08,7.01,7.57,1.69,8.42,3.89 \text {, } \\
46.64\end{array}$ \\
\hline & $366 \mathrm{~nm}$ & 6 & $0.23,0.30,0.35,0.41,0.78,0.88$ & $18.66,8.93,10.36,4.77,39.22,8.05$ \\
\hline \multirow{2}{*}{4} & $254 \mathrm{~nm}$ & 9 & $0.11,0.24,0.26,0.44,0.52,0.64,0.70,0.78,0.93$ & $3.62,2.10,5.24,6.61,13.66,4.03,3.82,7.25,53,66$ \\
\hline & $366 \mathrm{~nm}$ & 5 & $0.23,0.31,0.46,0.77,0.87$ & $19.71,5.09,6.01,48.81,20.38$ \\
\hline \multirow{2}{*}{5} & $254 \mathrm{~nm}$ & 10 & $0.11,0.26,0.39,0.46,0.54,0.58,0.71,0.78,0.81,0.93$ & $5.40,6.07,10.45,5.47,7.37,7.40,3.17,2.80,3.58,48.29$ \\
\hline & $366 \mathrm{~nm}$ & 4 & $0.24,0.47,0.78,0.87$ & $13.74,6.97,54.98,24.31$ \\
\hline \multirow{2}{*}{6} & $254 \mathrm{~nm}$ & 8 & $0.18,0.26,0.38,0.45,0.52,0.58,0.77,0.94$ & $2 / 81,11.26,3.00,4.28,15.63,9.26,14.26,39.51$ \\
\hline & $366 \mathrm{~nm}$ & 6 & $0.23,0.32,0.46,0.54,0.77,0.88$ & $21.00,4.31,8.11,2.81,51.05,12.73$ \\
\hline \multirow{2}{*}{7} & $254 \mathrm{~nm}$ & 9 & $0.24,0.34,0.44,0.53,0.64,0.71,0.78,0.89,0.94$ & $13.31,4.66,6.14,17.59,3.23,5.39,8.56,3.70,37.40$ \\
\hline & $366 \mathrm{~nm}$ & 3 & $0.24,0.78,0.88$ & $18.19,60.26,21.55$ \\
\hline \multirow{2}{*}{8} & $254 \mathrm{~nm}$ & 9 & $0.10,0.25,0.40,0.55,0.64,0.70,0.79,0.94$ & $2.78,8.13,2.85,4.85,15.51,6.71,6.64,8.95,43,58$ \\
\hline & $366 \mathrm{~nm}$ & 5 & $0.24,0.32,0.49,0.77,0.88$ & $16.98,6.45,5.85,53.57,17.15$ \\
\hline \multirow{2}{*}{9} & $254 \mathrm{~nm}$ & 8 & $0.25,0.43,0.49,0.53,0.65,0.71,0.78,0.94$ & $9.25,5.50,2.41,21.23,2.94,3.66,9.55,45.46$ \\
\hline & $366 \mathrm{~nm}$ & 5 & $0.25,0.32,0.48,0.78,0.88$ & $16.42,8.19,4.70,55.23,15.47$ \\
\hline \multirow{2}{*}{10} & $254 \mathrm{~nm}$ & 10 & $0.11,0.25,0.37,0.44,0.54,0.64,0.76,0.79,0.82,0.94$ & $3.99,9.43,4.21,8.80,20.48,3.69,2.33,3.77,6.44,36.86$ \\
\hline & $366 \mathrm{~nm}$ & 3 & $0.24,0.77,0.88$ & $17.70,55.54,26.77$ \\
\hline \multirow{2}{*}{11} & $254 \mathrm{~nm}$ & 8 & $0.28,0.36,0.45,0.54,0.64,0.72,0.77,0.94$ & $9.38,7.70,10.47,24.05,3.75,3.80,11.04,29.83$ \\
\hline & $366 \mathrm{~nm}$ & 4 & $0.26,0.34,0.77,0.89$ & $16.42,9.61,59.53,14.44$ \\
\hline
\end{tabular}


Table 3: Con

\begin{tabular}{|c|c|c|c|c|}
\hline \multirow{2}{*}{12} & $254 \mathrm{~nm}$ & 8 & $0.13,0.26,0.45,0.54 .0 .64,0.71,0.79,0.93$ & $2.28,8.52,9.07,17.72,3.66,4.42,11.08,43.25$ \\
\hline & $366 \mathrm{~nm}$ & 5 & $0.24,0.33,0.47,0.77,0.88$ & $18.47,7.24,7.69,52.81,14.42$ \\
\hline \multirow{2}{*}{13} & $254 \mathrm{~nm}$ & 5 & $0.26,0.54,0.79,0.83,0.94$ & $10.98,22.16,8.25,2.12,56.48$ \\
\hline & $366 \mathrm{~nm}$ & 4 & $0.25,0.33 .0 .78,0.89$ & $18.94,9.97,51.23,19.85$ \\
\hline \multirow{2}{*}{14} & $254 \mathrm{~nm}$ & 7 & $0.28,0.45,0.55,0.65,0.71,0.78,0.93$ & $8.84,8.44,19.37,2.91,3.20,9.31,47.92$ \\
\hline & $366 \mathrm{~nm}$ & 4 & $0.26,0.34,0.77,0.88$ & $19.09,9.76,57.47,13.68$ \\
\hline \multirow{2}{*}{15} & $254 \mathrm{~nm}$ & 8 & $0.26,0.39,0.45,0.55,0.63 .0 .71,0.79,0.93$ & $10.20,1.61,5.88,21.58,3.20,2.26,7.97,46.30$ \\
\hline & $366 \mathrm{~nm}$ & 5 & $0.26,0.34,0.48,0.77,0.88$ & $20.37,10.99,3.22,52.13,13.29$ \\
\hline \multirow[t]{2}{*}{16} & $254 \mathrm{~nm}$ & 13 & $\begin{array}{c}0.10,0.20,0.27,0 / 32,0.39,0.44,0.55,0.58,0.63,0.65 \\
0.71,0.77,0.93\end{array}$ & $\begin{array}{c}6.06,2.43,6.88,1.68,1.96,3.77,6.40,9.84,0.47,3.75,2.95 \\
11.40,42.41\end{array}$ \\
\hline & $366 \mathrm{~nm}$ & 5 & $0.25,0.33 .0 .57,0.76,0.88$ & $16.70,7.96,1.68,59.08,14.58$ \\
\hline \multirow{2}{*}{17} & $254 \mathrm{~nm}$ & 9 & $0.11,0.17,0.27,0.46,0.55,0.64,0.70,0.77,0.92$ & $2.99,1.62,8.20,3.65,11.16,2.57,5.83,10.13,53.85$ \\
\hline & $366 \mathrm{~nm}$ & 5 & $0.24,0.33,0.55,0.77,0.87$ & $21.90,7.96,3.86,52.25,14.03$ \\
\hline \multirow{2}{*}{18} & $254 \mathrm{~nm}$ & 9 & $0.11,0.21,0.26,0.45,0.54,0.64,0.71,0.79,0.92$ & $2.34,3.30,7.12,3.91,13.48,4.80,4.96,9.86,50.23$ \\
\hline & $366 \mathrm{~nm}$ & 6 & $0.25,0.34,0.53,0.66,0.77,0.87$ & $19.64,5.52,5.86,3,65,53.43,11.89$ \\
\hline \multirow[t]{2}{*}{19} & $254 \mathrm{~nm}$ & 12 & $\begin{array}{c}0.11,0.20,0.27,0.36,0.46,0.54,0.64,0.67,0.71,0.75 \\
0.79,0.92\end{array}$ & $\begin{array}{c}4.70,2.38,9.51,4.40,2.63,8.69,1.71,2.18,3.52,0.56,7.00, \\
52.74\end{array}$ \\
\hline & $366 \mathrm{~nm}$ & 5 & $0.24,0.33,0.77,0.87,0.93$ & $21.84,7.19,47.75,18.85,4.38$ \\
\hline \multirow{2}{*}{20} & $254 \mathrm{~nm}$ & 8 & $0.10,0.15,0.23,0.27,0.52,0.65,0.71,0.78$ & $8.70,14.28,5.20,15.75,29.31,9.04,6.69,11.02$ \\
\hline & $366 \mathrm{~nm}$ & 5 & $0.14,0.23,0.77,0.87,0.92$ & $5.10,26.30,44.55,10.01,14.04$ \\
\hline \multirow{2}{*}{21} & $254 \mathrm{~nm}$ & 10 & $0.11,0.22,0.26,0.31,0.46,0.50,0.52,0.59,0.66,0.73$ & $1.84,3.84,14.64,15.37,11.64,7.77,3.63,16.35,17.03,7.88$ \\
\hline & $366 \mathrm{~nm}$ & 5 & $0.24,0.34,0.50,0.78,0.86$ & $18.09,3.03,6.52,43.01,29.35$ \\
\hline \multirow{2}{*}{22} & $254 \mathrm{~nm}$ & 8 & $0.10,0.14,0.29,0.38,0.48,0.60,0.72,0.86$ & $1.42,1.64,5.99,2.89,5.45,7.57,3.08,71.96$ \\
\hline & $366 \mathrm{~nm}$ & 6 & $0.29,0.38,0.55,0.78,0.87,0.91$ & $19.72,8.51,9.65,36.21,7.51,18.40$ \\
\hline \multirow{2}{*}{23} & $254 \mathrm{~nm}$ & 9 & $0.11,0.28,0.34,0.42,0.50,0.61,0.72,0.75,0.88$ & $7.37,4.51,8.36,1.49,4.87,10,89,1.44,1.86,59.22$ \\
\hline & $366 \mathrm{~nm}$ & 8 & $0.28,0.35,0.41,0.56,0.60,0.78,0.88,0.92$ & $4.95,14.02,10.54,4.97,5.19,42.93,7.95,9.46$ \\
\hline \multirow{2}{*}{24} & $254 \mathrm{~nm}$ & 9 & $0.11,0.15,0.33,0.51,0.62,0.68,0.73,0.80,0.91$ & $2.94,2.12,18.72,4.85,11.31,6.08,5.07,4.63,44.27$ \\
\hline & $366 \mathrm{~nm}$ & 7 & $0.34,0.41,0.54,0.61,0.78,0.87,0.93$ & $23.40,10.78,3.68,4.10,46.46,9.51,2.06$ \\
\hline \multirow[t]{2}{*}{25} & $254 \mathrm{~nm}$ & 12 & $\begin{array}{c}0.19,0.22,0.28,0.36,0.51,0.56,0.60,0.64,0.68,0.73 \\
0.80,0.91\end{array}$ & $\begin{array}{c}3.00,3.95,1.85,4.83,6.66,10.01,5.20,7.52,2.90,2.75,3.14 \\
48.18\end{array}$ \\
\hline & $366 \mathrm{~nm}$ & 7 & $0.22,0.34,0.42,0.55,0.78,0.88,0.93$ & $2.82,15.34,7.70,8.15,50.86,6.32,8.82$ \\
\hline \multirow[t]{2}{*}{26} & $254 \mathrm{~nm}$ & 12 & $\begin{array}{c}0.12,0.14,0.19,0.23,0.8,0.35,0.48,0.51,0.63,0.79 \\
0.87,0.91\end{array}$ & $\begin{array}{c}2.22,2.04,1.23,3.95,2.86,7.73,2.60,2.17,6.15,5.46,3.00, \\
60.60\end{array}$ \\
\hline & $366 \mathrm{~nm}$ & 7 & $0.28,0.34,0.41,0.55,0.78,0.86,0.92$ & $2.62,14.41,7.97,3.26,44.13,20.26,7.34$ \\
\hline \multirow{2}{*}{27} & $254 \mathrm{~nm}$ & 10 & $0.11,0.24,029,0.34,0.50,0.57,0.63,0.72,0.87,0.91$ & $4.23,1.20,3.24,7.41,2.62,7.01,12.46,3.28,5.00,53.55$ \\
\hline & $366 \mathrm{~nm}$ & 8 & $0.34,0.40,0.54,0.59,0.61,0.78,0.87,0.93$ & $13.06,6.86,3.27,4.72,6.06,39.99,18.29,7.75$ \\
\hline \multirow{2}{*}{28} & $254 \mathrm{~nm}$ & 10 & $0.14,0.34,0.38,0.60,0.66,0.72,0.78,0.81,0.90$ & $1.22,6.61,2.75,7.18,2.73,3.51,0.61,1.83,2.37,71.18$ \\
\hline & $366 \mathrm{~nm}$ & 5 & $0.29,0.54,0.78,0.87,0.92$ & $23.37,5.73,48.31,16.22,6.37$ \\
\hline \multirow[t]{2}{*}{29} & $254 \mathrm{~nm}$ & 13 & $\begin{array}{c}0.11,0.14,0.22,0.30,0.39,0.42,0.48,0.54,0.60,0.66 \\
0.68,0.71,0.77\end{array}$ & $\begin{array}{c}6.40,3.32,1.77,14.29,4.88,2.51,7.47,6.21,20.31,6.17,1.54, \\
9.18,15.95\end{array}$ \\
\hline & $366 \mathrm{~nm}$ & 7 & $0.28,0.38,0.42,0.52,0.77,0.86,0.91$ & $17.97,6.77,1.76,8.32,42.73,14.30,8.15$ \\
\hline \multirow{2}{*}{30} & $254 \mathrm{~nm}$ & 10 & $0.08,0.14,0.27,0.36,0.33,0.54,0.59,0.65,0.70,0.76$ & $5.39,3.40,4.73,12.45,10.75,10.05,14.93,13.60,14.77$ \\
\hline & $366 \mathrm{~nm}$ & 5 & $0.27,0.35,0.71,0.76,0.86$ & $9.91,4.42,5.89,56.34,23.44$ \\
\hline
\end{tabular}

\section{ACKNOWLEDGEMENT}

This work supported by Directorate of Research and Community Engagement (DRPM) Universitas Indonesia via Hibah PITTA 2016

\section{CONFLICT OF INTEREST}

None

\section{ABBREVIATION USED}

[BMIM]Br: 1-butyl-3-methyl-imidazolium bromide; [BIM]BF4: 1-butyl-3-methyl-imidazolium tetrafluoroborate; [BMIM]C1OSO3: 1-butyl-3-methyl-imidazolium methylsulfate; [OMIM]Br: 1-octyl-3 methylimidazolium Bromide; [HMIM]Cl: 1-hexyl-3-methylimidazolium chloride; HRE: Heat Reflux Extraction; IL-MAE: Ionic liquid based 
microwave-assisted extraction; ILs: Ionic Liquids; HPTLC: High Performance Thin Layer Chromatography; TLC: Thin Layer Chromatography; P. pellucida: Peperomia pellucida (L) Kunth

\section{REFERENCES}

1. Espino M, Fernández MDLÁ, Gomez FJ V, Silva MF. Natural Designer Solvents for Greening Analytical Chemistry. Trends Anal Chem. 2015.

2. Jessop PG, Jessop DA, Fu D, Phan L. Solvatochromic parameters for solvents of interest in green chemistry. Green Chem. 2012;14(5):1245. https://doi. org/10.1039/c2gc16670d.

3. Du F, Xiao X, Li G. Application of ionic liquids in the microwave-assisted extraction of trans-resveratrol from Rhizoma Polygoni Cuspidati. J Chromatogr A. 2007;1140(1-2):56-62. https://doi.org/10.1016/j.chroma.2006.11.049 PMid:17141255.

4. Lu Y, Ma W, Hu R, Dai X, Pan Y, Ionic liquid-based microwave-assisted extraction of phenolic alkaloids from the medicinal plant Nelumbo nucifera Gaertn. J Chrom A. 2008;1208(1-2):42-6. https://doi.org/10.1016/j.chroma.2008.08.070 PMid:18774575

5. Cao $X, Y e X, L u Y, Y u Y, M o W$. Ionic liquid-based ultrasonic-assisted extraction of piperine from white pepper. Anal Chim Acta. 2009;640(1-2):47-51. https://doi. org/10.1016/j.aca.2009.03.029 PMid:19362618.

6. Han D, Row KH. Determination of luteolin and apigenin in celery using ultrasonic-assisted extraction based on the aqueous solution of ionic liquid coupled with HPLC quantification. J Sci Food Agric. 2011;91(15):2888-92. https://doi. org/10.1002/jsfa.4553 PMid:21748734

7. Lu C, Wang H, LvW, et al. lonic liquid-based ultrasonic/microwave-assisted extraction combined with UPLC-MS-MS for the determination of tannins in Galla chinensis. Nat Prod Res. 2012;26(19):1841-7. https://doi.org/10.1080/14786419. 2011.607454 PMid:21999458.

8. Zhang D-Y, Yao X-H, Duan M-H, et al. An effective negative pressure cavitationmicrowave assisted extraction for the determination of phenolic compounds in P. calliantha H. Andr. Analyst. 2013;138(16):4631. https://doi.org/10.1039/ c3an36534d PMid:23762895.

9. Zhang Y, Liu Z, LiY, Chi R. Optimization of Ionic liquid-based Microwave-assisted Extraction of Isoflavones from Radix Puerariae by Response Surface Methodology. Sep Purif Technol. 2014.

10. Zhang Q, Zhao S, Chen J, Zhang L. Application of ionic liquid-based microwave-assisted extraction of flavonoids from Scutellaria baicalensis Georgi. J Chromatogr B. 2015;1002:411-7. https://doi.org/10.1016/j.jchromb.2015.08.021 PMid:26367465.

11. Wang X, Cai C, Li X. Optimal Extraction of Gallic Acid from Suaeda glauca Bge. Leaves and Enhanced Efficiency by lonic Liquids. Int J Chem Eng. 2016;2016.

12. Heyne K. The Indonesia Usage Plants. Third Edition. Jakarta: Departement of Forestry, Yayasan Sarana Wana Jaya; 2007. (In Bahasa)

13. Hutahaean I. Effectivity of the ethyl acetate fraction from Suruhan (Peperomia pellucida L. Kunth) as ACE Inhibitory against Mice (Rattus novergicuss) 2KIC Goldblatt hypertension induced. Skripsi, Depok: Faculty of Pharmacy, Universitas Indonesia. 2015. (In Bahasa)

14. Ooi D, Iqbal S, Ismail M. Proximate Composition, Nutritional Attributes and Mineral Composition of Peperomia pellucida L. (Ketumpangan Air) Grown in Malaysia. Molecules. 2012;17(12):11139-45. https://doi.org/10.3390/molecules170911139 PMid:22986924.
15. Khan MR, Omoloso AD. Antibacterial activity of Hygrophila stricta and Peperomia pellucida. Fitoterapia. 2002;73(3):251-4. https://doi.org/10.1016/S0367326X(02)00066-7.

16. Mensah JK, Okoli RI, Turay AA. Phytochemical Analysis of Medicinal Plants Used for the Management of Hypertension by Esan people of Edo State, Nigeria. Ethnobot Leaf. 2009:13(12):73-87.

17. Gini TG, Jothi GJ. Preliminary Phytochemical Screening for Active Compounds in the Whole Plant Extract of Peperomia pellucida (Linn.) HBK (Piperaceae) and Marsilea quadrifolia Linn. (Marsileaceae). Int J Pharmacogn Phytochem Res. 2013;5(3):200-14.

18. Leena PK, Annam C. Preliminary Phytochemical Screening and In-vitro Cytotoxicity Activity of Peperomia pellucida Linn. Int J Compr Pharm. 2013;4(8):1-4.

19. Abere TA, Okpalaonyagu SO. Pharmacognostic Evaluation and Antisickling Activity of the Leaves of Peperomia pellucida (L.) HBK (Piperaceae). African J Pharm Pharmacol. 2015:9(11):367-74.

20. Verma RS, Padalia RC, Goswami P, Chauhan A. Essential oil composition of Peperomia pellucida (L.) Kunth from India. J Essent Oil Res. 2015;27(2):89-95 https://doi.org/10.1080/10412905.2014.982878.

21. Xu S, Li N, Ning M-M, Zhou C-H, Yang Q-R, Wang M-W. Bioactive Compounds from Peperomia pellucida. J Nat Prod. 2006;69(2):247-50. https://doi. org/10.1021/np050457s PMid:16499324.

22. Bayma JDC, Arruda MSP, Müller AH, Arruda AC, Canto WC. A dimeric ArC2 compound from Peperomia pellucida. Phytochemistry. 2000;55(7):779-82. https://doi.org/10.1016/S0031-9422(00)00224-7.

23. Khan A, Rahman M, Islam S. Isolation and Bioactivity of a Xanthone Glycoside from Peperomia pellucida. Life Sci Med Res. 2010;2010:1-10.

24. Rojas-Martínez R, Arrieta J, Cruz-Antonio L, Arrieta-Baez D, Velázquez-Méndez AM, Sánchez-Mendoza ME. Dillapiole, isolated from Peperomia pellucida, shows gastroprotector activity against ethanol-induced gastric lesions in Wistar rats. Molecules. 2013;18(9):11327-37. https://doi.org/10.3390/molecules180911327 PMid:24064453.

25. Susilawati Y, Nugraha R, Muhtadi A, Soetardjo S, Supratman U. (S)-2-Methyl-2(4-methylpent-3-enyl)-6-(propan-2-ylidene)-3,4,6,7-tetrahydropyrano[4,3-g]chromen-9(2H)-one. Molbank. 2015;2015(2): M855. https://doi.org/10.3390/M855.

26. Kurniawan A, Saputri FC, Ahmad I, Mun A. Isolation of Angiotensin Converting Enzyme (ACE) Inhibitory Activity Quercetin from Peperomia pellucida. Int J PharmTech Res. 2016;9(7):115-21.

27. Harborne JB. Metode Fitokimia. Bandung: Penerbit ITB; 1987. PMid:3691654.

28. Du F, Xiao X, Luo X, Li G. Application of ionic liquids in the microwave-assisted extraction of polyphenolic compounds from medicinal plants. Talanta. 2009;78:1177-84. https://doi.org/10.1016/j.talanta.2009.01.040 PMid:19269490.

29. Ma W, Lu Y, Hu R, Chen J, Zhang Z, Pan Y. Application of ionic liquids based microwave-assisted extraction of three alkaloids $\mathrm{N}$-nor nuciferine, O-nor nuciferine, and nuciferine from Lotus leaf. Talanta. 2010;80(3):1292-7. https://doi. org/10.1016/j.talanta.2009.09.027 PMid:20006090.

30. LiuT, Sui X, Zhang R, Yang L, ZuY, Zhang L. Application of ionic liquids based microwave-assisted simultaneous extraction of carnosic acid, rosmarinic acid and essential oil from Rosmarinus officinalis. J Chromatogr A. 2011:1218(47):84809. https://doi.org/10.1016/j.chroma.2011.09.073 PMid:21999917.

31. Xu W, Chu K, Li H, et al. Ionic Liquid-Based Microwave-Assisted Extraction of Flavonoids from Bauhinia championii (Benth.) Benth. Molecules. 2012;17(12):14323-35. https://doi.org/10.3390/molecules171214323 PMid:23208467.

\section{ABOUT AUTHORS}

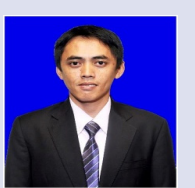

Islamudin Ahmad, a doctoral student at Departement of Pharmaceutical Sciences, Faculty of Pharmacy, Universitas Indonesia (UI) Depok, West Java, Indonesia. And also as a lecturer at Faculty of Pharmacy, Mulawarman University The doctoral research focused on the study of angiotensin converting enzyme (ACE) inhibitory active compound from the natural product for drugs discovery as antihypertension.

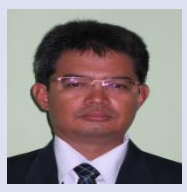

Abdul Mun'im, Ph.D., Associate Professor at Departement Pharmaceutical Sciences, Faculty of Pharmacy, Universitas Indonesia (UI) Depok, West Java, Indonesia. He has experience in the area of Pharmacognosy and Phytochemistry, working in drugs discovery of herbal plants, extraction technology, Metabolite profiling, Structure Elucidation, and Degenerative Diseases (such as diabetes mellitus, antihypertension, and cholesterol).

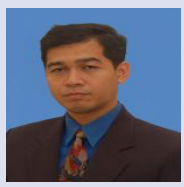

Arry Yanura: Is assosiate Professor from Department of Biomedical Computation, Faculty of Pharmacy, Universitas Indonesia. He holds PhD from Nara Institute of Science and Technology (NAIST), Japan, from Structural Biology/protein crystallography laboratory. In 2002, he visited National Institute of Health (NIH), Bethesda, USA. 


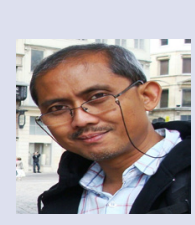

Kamarza Mulia: Is Assosiate professor from Department of Chemical Enginering, Faculty of Engginering, Universitas Indoensia. His primary research focuc is controlled release drugs and bioactive compound from biodegredable and biocompatible polymers. .

Cite this Article: Ahmad I, Yanuar A, Mulia K, Mun'im A. Application of lonic Liquid based Microwave-Assisted Extraction of the Secondary Metabolite from Peperomia pellucida (L) Kunth. Pharmacogn J., 2017:9(2):227-34. 